\title{
RECOMMENDATIONS FOR SPIRAL PULMONARY ARTERIOPLASTY
}

\author{
Reshma Biniwale ${ }^{1}$ \\ ${ }^{1}$ University of California Los Angeles Department of Surgery
}

December 18, 2021

\begin{abstract}
Case Video Summary: This video demonstrates the principle of spiral PA plasty whereby the length of branch pulmonary arteries can be increased simultaneous to the increase in diameter by using a patch that is anastomosed spirally around the vessels.
\end{abstract}

\section{RECOMMENDATIONS FOR SPIRAL PULMONARY ARTERIOPLASTY}

Reshma Biniwale MD1

1: University of California at Los Angeles, Department of Surgery, Division of Cardiac Surgery

The author reports no Conflicts of interest

IACUC approved Tissue sharing protocol 7/31/2019

Funding is provided by the Rachel Cooper Foundation Congenital Cardiac Research Fund

IRB approval N/A since it is an animal tissue study,

Consent statement N/A since it is an animal tissue study

Clinical trial registration is not applicable for our study.

Corresponding author:

Reshma Biniwale MD

10833 Le Conte Avenue, Suite 62-266 CHS, Los Angeles CA 90095

Email: rbiniwale@mednet.ucla.edu

Phone: (310) 206-5900 Fax: (310) 2065901

Objective: Branch pulmonary artery stenosis is a persistent problem following LeCompte maneuver of the pulmonary arteries (PAs) in arterial switch operations as well as root translocation. Our technique allows for elongation of the branch PAs as well as enlargement, decreasing the narrowing that results from stretch.

\section{Case Video Summary:}

This video demonstrates the principle of spiral PA plasty whereby the length of branch pulmonary arteries can be increased simultaneous to the increase in diameter by using a patch that is anastomosed spirally around the vessels.

In a porcine heart lung block, the ascending aorta is transected in preparation for the "Lecompte maneuver". 
The pulmonary arteries have been translocated anteriorly after extensive mobilization up to the hilar branches.

The incision in the Pulmonary arteries is started posteriorly to simulate the defects in the posterior sinuses left after coring out of the buttons in an arterial switch procedure.

The spiral incision is taken cephalad and then brought anteroinferiorly to the level of the hilum.

Now a PTFE patch is sutured to the left PA. The suturing is accomplished from hilum to the central pulmonary bifurcation. This increases the diameter of the LPA after patching.

A similar incision is made over the right pulmonary artery and a second patch sutured in a spiral fashion. In clinical practice a patch made of autologous pericardium or homograft is preferable to have tissue handling similar to native pulmonary artery tissue.

You can observe that the width of the patch correlates to the increase in the length of the pulmonary branches and that the length of the patch corresponds to the increase in the diameter of the pulmonary arteries.

The final result of the patch plasty is demonstrated with greatly enlarged diameter as well as length. The size of the patch can be tailored to the needs of the patient.

Now the main pulmonary artery is anastomosed to the pulmonary artery bifurcation in a manner similar to that done after arterial switch taking care to maintain the increased dimensions after the branch PA plasty.

The Right pulmonary artery shows the results of a more spiral incision as compared to the left pulmonary artery which has a more traditional incision.

Conclusions: This technique is important clinically for surgical management of branch PA stenosis following LeCompte maneuver. The elongation of the artery corresponds to the width of the patch and the enlargement of the diameter corresponds to the length of the patch.

TABLE 1

\begin{tabular}{llll}
\hline ANIMAL & & PRE $\mathrm{mm}$ & POST $\mathrm{mm}$ \\
\hline PIG1 & LPA length & $14.7+12.0$ & 34.9 \\
6116877 & LPA diameter & 8.46 & 17.7 \\
& RPA length & 13.29 & 34.4 \\
& RPA diameter & 8.66 & 14.1 \\
& MPA length & 28.8 & 34.3 \\
PIG 2 & MPA diameter & 13.8 & 12.5 \\
6174516 & LPA length & $12.01+14.76$ & $15.71+12.24$ \\
& LPA diameter & 8.46 & 15.71 \\
& RPA length & 13.29 & 16.4 \\
& RPA diameter & 8.66 & 20.18 \\
& MPA length & 14.1 & 24.0 \\
& MPA diameter & 14.9 & 18.0 \\
\hline
\end{tabular}

FIGURE 1: Spiral pulmonary arterioplasty diagram 


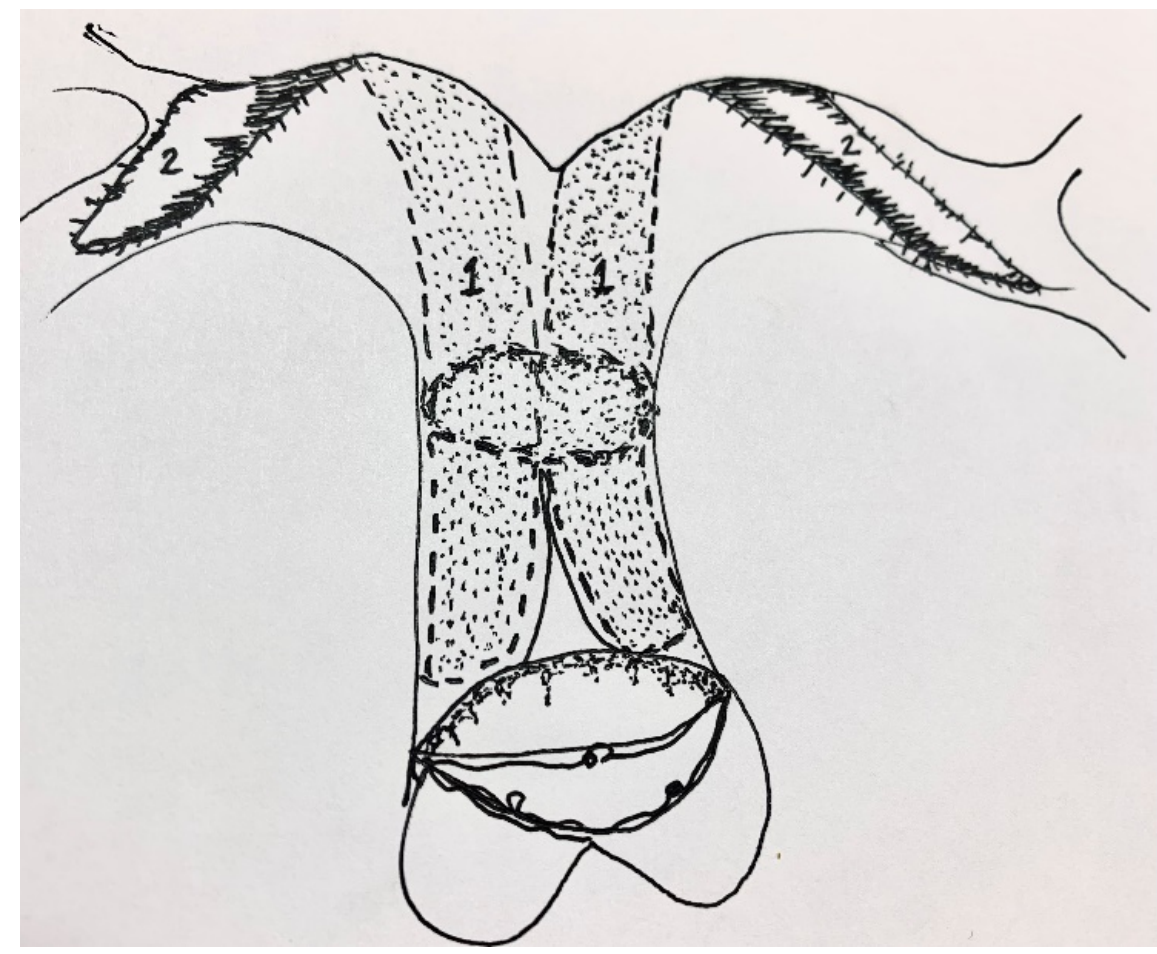

1: Posterior patch augmentation

2. Anterior patch continuation

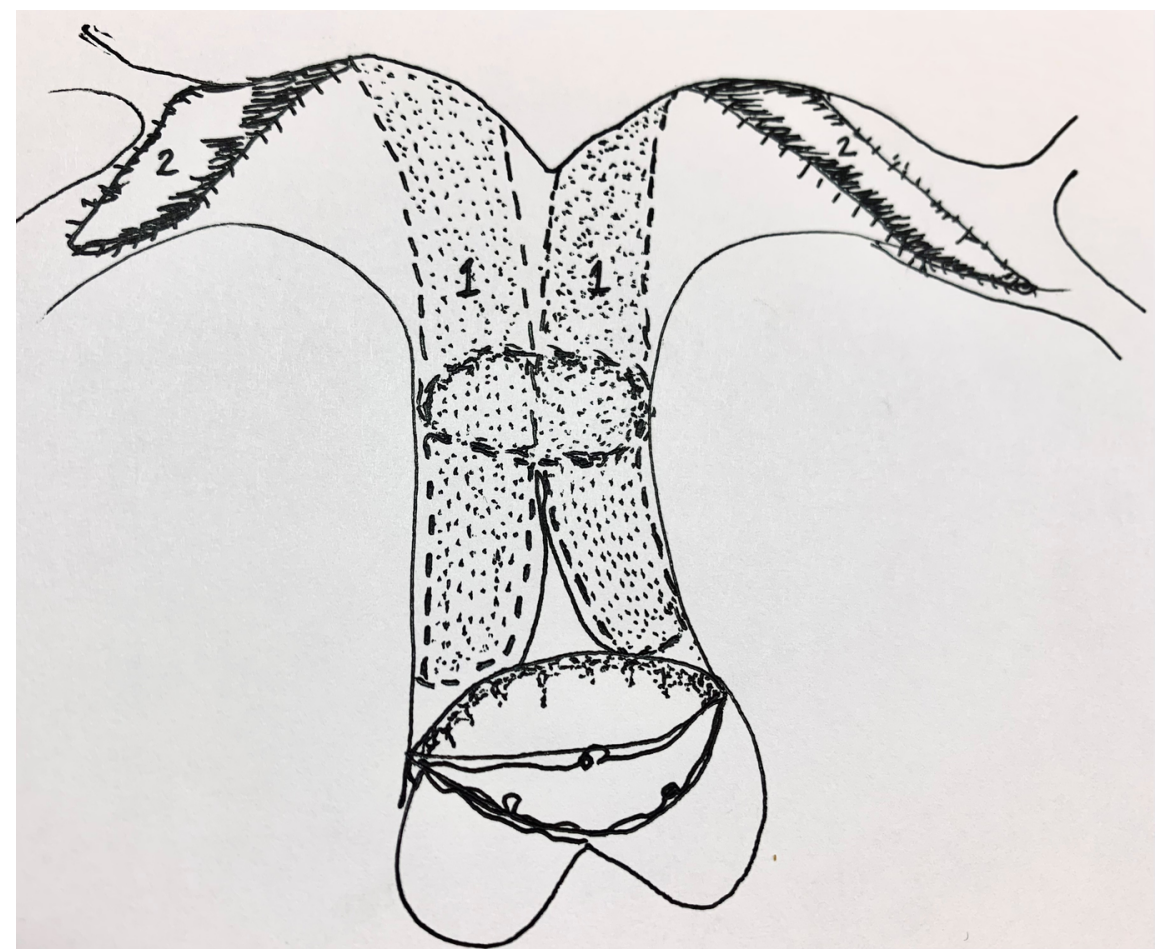




\section{Hosted file}

TABLE 1.docx available at https://authorea.com/users/451742/articles/549932-recommendationsfor-spiral-pulmonary-arterioplasty 